Cahiers de recherches médiévales

Journal of medieval studies

$8 \mid 2001$

La protection spirituelle au Moyen Âge

\title{
Saint Olaf
}

Un saint protecteur norvégien à la fin du Moyen Âge

\section{Stéphane Coviaux}

\section{(2) OpenEdition}

Journals

Édition électronique

URL : https://journals.openedition.org/crm/384

DOI : $10.4000 / \mathrm{crm} .384$

ISSN : 1955-2424

Éditeur

Honoré Champion

Édition imprimée

Date de publication : 15 janvier 2001

Pagination : 57-72

ISSN : 1272-9752

Référence électronique

Stéphane Coviaux, «Saint Olaf », Cahiers de recherches médiévales [En ligne], 8 | 2001, mis en ligne le 13 mars 2008, consulté le 15 décembre 2022. URL : http://journals.openedition.org/crm/384 ; DOI : https://doi.org/10.4000/crm.384

Ce document a été généré automatiquement le 15 décembre 2022.

Tous droits réservés 


\title{
Saint Olaf
}

Un saint protecteur norvégien à la fin du Moyen Âge

\author{
Stéphane Coviaux
}

Dans son ouvrage intitulé Rassurer et protéger, publié en 1989, Jean Delumeau faisait du culte de la Vierge et des saints un des éléments fondamentaux d'un système de protection dont l'objet était de satisfaire le besoin de sécurité des hommes de la fin du Moyen Âge et de l'époque moderne en Occident, en apaisant leurs peurs et leurs angoisses ${ }^{1}$. Elles étaient certainement pour partie liées à la perspective de la mort et de l'au-delà, et l'incertitude dans laquelle les chrétiens étaient quant au sort réservé alors à leur âme pouvait générer la recherche d'une protection spirituelle auprès des saints, ces intercesseurs anciens et traditionnels. La question se pose de savoir à partir de quand ils recherchèrent auprès d'eux ce type de protection.

2 L'étude d'un cas particulier, en l'occurrence celui de saint Olaf, pourra peut-être fournir quelques éléments de réponse à cette question délicate. On sait comment le roi Olaf Haraldsson, mort en 1030 alors qu'il tentait de reprendre possession de la Norvège, sur laquelle il avait régné entre 1015 et 1028, fut en l'espace de quelques années seulement considéré comme saint et martyr d'abord en son propre royaume, puis dans la totalité du monde nordique, de l'Angleterre à l'île de Gotland ${ }^{2}$. On retrouve d'ailleurs un écho de cette précoce renommée vers 1075 sous la plume du chanoine Adam de Brême, dans la «Description des îles du Nord» qui clôt les Gesta Hammaburgensis ecclesice pontificum: Nidaros, l'actuelle ville de Trondheim, qui abritait les reliques de saint Olaf, attirait dès cette époque des pèlerins venus de régions très lointaines dans l'espoir d'une guérison ${ }^{3}$. Rien n'indique que sa réputation ait eu à souffrir à la fin du Moyen Âge de l'érosion du temps ou de la concurrence de nouveaux saints nordiques comme sainte Brigitte. Bien au contraire, il resta jusqu'au temps de la Réforme le principal saint de Scandinavie, celui que l'on invoquait le plus volontiers dans l'espoir d'une guérison ou quand on se sentait menacé par un danger quelconque ; la popularité de son culte ne se démentit nullement alors dans l'ensemble des régions septentrionales de l'Europe ${ }^{4}$.

3 Cette étude n'aura cependant pas pour ambition d'en donner une description pour les derniers siècles du Moyen Âge, mais plutôt d'examiner quelle protection les Norvégiens 
attendaient alors de leur saint roi. Son objectif ultime sera de déterminer à partir de quand on peut supposer qu'ils se mirent à rechercher auprès de lui une protection non seulement contre les dangers qui menaçaient leurs biens ou même leur vie ici-bas, mais aussi pour le salut de leur âme, autrement dit une protection spirituelle.

Il est relativement facile de déterminer quelle image de saint Olaf l'Église voulut donner aux Norvégiens des derniers siècles du Moyen Âge, et en particulier quelle protection elle les incitait à rechercher auprès de lui. Plusieurs sources ecclésiastiques permettent en effet de connaître la légende de saint Olaf telle qu'elle a été forgée à Nidaros au cours de la seconde moitié du XII ${ }^{e}$ siècle, légende qui ne semble guère avoir varié par la suite. Mais il parait judicieux de se demander dans quelle mesure l'image qui s'en dégage correspondait précisément aux attentes des Norvégiens. Pour déterminer en particulier quelle protection ils pouvaient attendre de lui, il faut donc avoir recours à des sources que faute de mieux nous qualifierons de profanes. Entendons par là, outre quelques sagas royales rédigées en Islande et en Norvège à partir de la fin du XII ${ }^{e}$ siècle, et qui sont par conséquent à peu près contemporaines de la cristallisation de la légende ecclésiastique de saint olaf, les statuts de guildes norvégiennes placées sous son patronage. Comme ces statuts datent du XIV siècle, ils permettent de traquer une éventuelle évolution des attentes des Norvégiens de la fin du Moyen Âge et, puisque telle est notre ambition, de voir s'ils en vinrent ou non à attendre une protection spirituelle de la figure familière et bienveillante du plus ancien des saints rois scandinaves.

\section{L'Église de Norvège et l'image de saint Olaf (fin XIIe-début XIII siècle)}

5 En 1152-1153 le légat pontifical Nicolas Breakspear, le futur Adrien IV, se rendit dans le Nord pour y fonder au nom du pape Eugène III la province ecclésiastique de Nidaros, couronnant ainsi le développement de l'Église norvégienne depuis le début du $\mathrm{XI}^{e}$ siècle. Celle-ci se trouvait désormais coupée de la métropole de Lund, née vers 1103, et regroupait onze diocèses couvrant la Norvège et les îles de l'Atlantique Nord ${ }^{5}$. À l'occasion de la visite du légat furent également introduites en Norvège d'importantes réformes ecclésiastiques, au nombre desquelles figurait la création de chapitres cathédraux chargés comme ailleurs en Europe de procéder à l'élection des évêques.

$\mathrm{Au}$ cours des années qui suivirent, la légende ecclésiastique de saint Olaf atteignit son plein développement en Norvège, à l'initiative du second archevêque de Nidaros, Eysteinn Erlendsson (1161-1188). Son pontificat constitua une étape majeure dans le développement de la jeune Église norvégienne ainsi que dans l'évolution politique du royaume, qui était depuis la mort du roi Sigurd Jorsalfar en 1130 le théâtre d'incessantes luttes entre prétendants à la couronne. Dans ce contexte troublé, l'archevêque voulut jouer un rôle de pacificateur. Prenant fait et cause pour l'un des compétiteurs, le jeune Magnus Erlingsson, il parvint à créer les conditions d'un retour à la stabilité en modernisant les structures politiques de la Norvège. Revenant en particulier sur les anciennes coutumes qui réglaient la succession royale et imposaient le partage du royaume entre les fils du roi défunt, il fit triompher le principe de la primogéniture masculine; il obtint par ailleurs de Magnus, qu'il couronna solennellement en 1163 , un serment par lequel le jeune roi s'engageait à respecter les 
libertés de l'Église, ainsi qu'une lettre de privilèges dans laquelle il déclarait tenir son royaume en fief de saint Olaf, rex perpetuus Norvegice.

7 C'est donc au moins en partie pour pacifier la Norvège que l'archevêque se fit le chantre du saint roi mort en 1030. Il le présenta comme l'archétype nordique du rex iustus et fit de lui un modèle auquel les rois étaient désormais invités à se conformer. Alors qu'il existait probablement déjà à Nidaros un récit de la translation des reliques de saint Olaf, qui n'a d'ailleurs pas été conservé, il composa lui-même en latin un bref récit de sa vie et de sa mort, la Passio beati Olavi, et il y adjoignit une collection de miracles dont un petit nombre seulement remontait à la période antérieure au milieu du XII ${ }^{e}$ siècle 6 . La plupart d'entre eux dataient en effet de son propre pontificat ou des années qui l'avaient immédiatement précédé. L'ensemble, désigné sous le titre Passio et miracula beati Olavi, a été conservé en deux versions. La plus ancienne est une version courte, qui fit l'objet d'une large diffusion dans tout le nord de l'Europe, mais dont nous n'avons pas conservé de manuscrit norvégien? ${ }^{7}$. D'autre part un manuscrit anglais provenant du monastère cistercien de Fountains et daté approximativement de 1200 nous a transmis une version longue de la même œuvre, qui ne comprend pas moins de cinquante miracles attribués au saint ${ }^{8}$. Selon les philologues, elle est bien l'œuvre du seul Eysteinn'. Il n'est d'ailleurs pas impossible qu'il l'ait achevée en Angleterre, où il dut s'exiler au début des années 1180 car il était alors en butte à l'hostilité du roi Sverre (1177-1202), qui était parvenu à s'imposer aux dépens de Magnus Erlingsson et qui entendait revenir sur les libertés de l'Église de Norvège ${ }^{10}$.

On ne saurait enfin oublier dans cette évocation des sources permettant de retracer le développement de la légende ecclésiastique de saint Olaf l'existence d'un homiliaire que l'on surnomme communément l'homiliaire vieux-norvégien (Gammel norsk homiliebok), transmis par un manuscrit datant du début du XIII ${ }^{\mathrm{e}}$ siècle ${ }^{11}$. Outre des homélies traduites d'Alcuin ou encore largement inspirées de Césaire d'Arles, ce recueil contient des pièces originales, dont une vie de saint olaf en langue vernaculaire et destinée à être lue lors de sa fête, le 29 juillet. Pour être très proche de la Passio Olavi qu'Eysteinn avait rédigée en latin, elle n'en constitue peut-être pas cependant la traduction directe ${ }^{12}$. Suit une collection de vingt miracles, regroupés sous la rubrique "Her segir fra jartcinum hins hoelga Olafs konungs", qui sont les vingt premiers miracles consignés par l'archevêque dans sa propre collection ${ }^{13}$.

9 Sur ces vingt miracles, neuf sont des guérisons; une telle proportion se retrouve d'ailleurs dans la version longue de la Passio et miracula beati Olavi. Saint Olaf aurait ainsi guéri des aveugles, des muets, des paralytiques, et serait également venu au secours de personnes qui, à l'instar de l'archevêque, avaient été victimes d'accidents. Eysteinn raconte en effet comment il bénéficia lui-même de la bienveillance du saint après avoir été grièvement blessé à la jambe lors des travaux de reconstruction de la cathédrale de Nidaros qu'il avait ordonnés ${ }^{14}$. Comme lui, tous les chrétiens pouvaient espérer en l'intercession de saint olaf pour obtenir une guérison, qu'ils soient Norvégiens ou étrangers, hommes ou femmes, humbles ou puissants ${ }^{15}$. Le saint roi de Norvège fut ainsi d'abord conçu comme un saint thaumaturge, et l'espoir de guérir était selon toute apparence la raison première qui poussait les pèlerins à se rendre auprès de ses reliques dès le $\mathrm{XI}^{\mathrm{e}}$ siècle.

10 Cependant on notera avec intérêt que, tant dans la Passio Olavi que dans l'homiliaire, ces miracles de guérison occupent une place plutôt restreinte par rapport à ce que l'on a pu constater pour d'autres saints et en d'autres contrées ${ }^{16}$. C'est que, selon l'Église 
norvégienne de la fin du XII siècle, saint Olaf déployait de bien d'autres manières son action bénéfique sur les habitants de son ancien royaume, et plus généralement sur tous ceux qui l'invoquaient ${ }^{17}$. Quelques exemples tirés de la Passio Olavi permettront d'avoir une idée de l'ampleur de son champ d'intervention. Deux récits racontent ainsi comment il tira des geôles païennes des prisonniers chrétiens, norvégiens et danois ${ }^{18}$; il fournit également des pierres aux chrétiens du Telemark, désireux de lui construire une église ${ }^{19}$; il retrouva un enfant perdu par ses parents ${ }^{20}$; il libéra des tentations de la chair un diacre danois, etc ${ }^{21}$. Par ailleurs il savait aussi châtier ceux qui lui manquaient de respect. La Passio Olavi et l'homiliaire racontent ainsi comment il punit un comte danois irreligieux, qui avait forcé sa pieuse servante norvégienne à cuire du pain le jour de sa fête. Une fois cuit, le pain se transforma en pierre ${ }^{22}$.

11 À la lecture de ces sources de la deuxième moitié du XII ${ }^{e}$ siècle, saint Olaf fait donc figure de saint aux compétences quasi universelles. En particulier, il apparaît comme un protecteur dont l'aide pouvait être recherchée en des occasions fort diverses. Il protégeait les hommes qui l'appellaient au secours contre toute forme d'injustice et en particulier contre les erreurs de la justice humaine. Dans un des récits les plus développés de la Passio Olavi et de l'homiliaire, on le voit ainsi secourir un paysan norvégien, condamné à la pendaison pour un crime dont il était pourtant innocent ${ }^{23}$. Il guérit également de ses blessures un prêtre anglais, qui avait été atrocement mutilé par deux Norvégiens qui le suspectaient à tort d'avoir courtisé leur sœur ${ }^{24}$. Dans un même ordre d'idées, il faisait bénéficier de sa protection les combattants dont la cause était juste, en particulier ceux qui combattaient contre les païens. L'existence d'une église Saint-Olaf à Constantinople s'expliquerait ainsi par l'aide qu'il aurait jadis accordée à un empereur byzantin l'ayant invoqué au cours d'un difficile combat contre des païens ; cette invocation aurait suffi à les faire fuir et à accorder la victoire au prince chrétien ${ }^{25}$. Par ailleurs, son intervention était jugée efficace contre toutes sortes de dangers. À quatre reprises dans la version longue de la Passio et miracula beati Olavi on le voit ainsi éteindre des incendies qui menaçaient soit des villes comme Nidaros ou Novgorod, soit des fermes norvégiennes ${ }^{26}$. Il sauva également de la noyade des enfants jouant imprudemment au bord d'une rivière, et protégea des marins surpris par la tempête et échoués sur un écueil ${ }^{27}$.

Deux miracles contés dans la Passio Olavi montrent que sa protection s'étendait aussi aux âmes des chrétiens menacées par les menées du diable, ce qui confère à cette protection une nette dimension spirituelle. Le plus intéressant dans cette optique est certainement le dix-septième miracle de la collection, qui est précédé d'un prologue dont il sera question plus loin, et qui se trouve assez fidèlement traduit dans l'homiliaire du début du XIII ${ }^{\mathrm{e}}$ siècle ${ }^{28}$. Son bénéficiaire fut un jeune homme d'Ytterøy, près de Nidaros, dont on apprend qu'il était aveuglé par le diable à un point tel qu'il en oubliait les châtiments à venir et courait à sa perte. Arriva la fête de saint Olaf, à l'occasion de laquelle il se rendit à Nidaros au milieu d'une foule de pèlerins. Lorsque les reliques du saint roi furent solennellement portées en procession et déposées devant l'église cathédrale, il commença à prendre conscience à la fois de la gloire du martyr et de la triste situation dans laquelle il se trouvait lui-même. Lorsque l'on souleva à nouveau la châsse pour la remporter, il se rendit à l'endroit où elle avait été déposée, se mit à gémir et à supplier saint Olaf de lui épargner le châtiment auquel il était promis. Sa prière fut entendue grâce à l'intercession du saint, et il sentit peu à peu son âme reprendre la force qu'elle avait perdue ; mais il ne voulut cependant pas faire 
pénitence. Alors Dieu envoya sur lui une grave maladie, dont il ne se remit pas avant de s'être entièrement repenti de ses fautes envers le Créateur.

L'autre miracle, que l'on ne lit à la différence du précédent que dans la version longue de la Passio Olavi, conte les mésaventures d'un jeune homme du Hålogaland, cette région située très au nord de la Norvège. S'étant rendu en forêt pour y couper du bois, il rencontra deux femmes d'une beauté si exceptionnelle qu'il les suivit sans réfléchir le moins du monde. Ils arrivèrent ainsi à une grotte, où se tenait un grand banquet, présidé par un homme dont on comprend qu'il n'était autre que le diable. Convié à prendre place et à participer aux agapes, le jeune homme était prêt à succomber à la tentation, quand, se souvenant de sa foi chrétienne, il invoqua Dieu et saint Olaf, ce qui lui donna la force de résister. Alors la grotte somptueuse se transforma en un lieu de feu et de terreur, peuplé de créatures monstrueuses. Menacé de toutes parts, le jeune homme s'en remit à la miséricorde divine; aussitôt une grande lumière dissipa les ténèbres et chassa les démons. Saint Olaf lui apparut alors et se présenta à lui comme le responsable de ce miracle ${ }^{29}$.

14 Ces deux récits dessinent donc les contours d'une protection spirituelle accordée par saint Olaf aux hommes menacés par le diable et par ses tentations. Dans les deux cas, ce ne sont ni l'intégrité matérielle ni l'intégrité physique des chrétiens qui sont en jeu, mais bien en définitive le salut de leur âme. Ce qui n'est qu'implicite dans le deuxième miracle, dont on ignore d'ailleurs s'il fit jamais l'objet d'une diffusion en Norvège, est totalement explicite en revanche dans le premier. Le malheureux jeune homme qui était prisonnier du diable, ou, comme on le lit dans le texte de l'homiliaire, était sous l'emprise d'un « poison mortel » (ban-vcenum drycc), en avait perdu de vue « les tortures de l'autre monde » (annars hæims pinslir) ${ }^{30}$.

C'était donc bien une protection universelle, à la fois matérielle et spirituelle, que l'Église de Norvège incitait les fidèles à rechercher auprès de la figure bienveillante de saint Olaf dès la fin du XII ${ }^{e}$ siècle. Et il est patent que cette fonction de protection leur fut constamment rappelée à la fin du Moyen Âge.

On en trouve une preuve notamment dans la liturgie norvégienne de cette époque. Elaborée à partir de la deuxième moitié du XII ${ }^{e}$ siècle à l'initiative de l'archevêque Eysteinn, la fête de saint Olaf, qui trouva sa forme définitive au début du XIII ${ }^{\mathrm{e}}$ siècle, fut un des temps forts de la vie religieuse des Norvégiens jusqu'à la Réforme ${ }^{31}$. Un certain nombre de bréviaires nordiques datant en règle générale $d u X^{e}$ siècle, ainsi que le Breviarium Nidrosiense, imprimé à Paris en 1519, permettent d'en connaître le déroulement ${ }^{32}$. Ces festivités très solennelles commençaient le 28 juillet à vêpres, se poursuivaient le lendemain par la célébration du martyre du saint, que la tradition fixe au 29 juillet 1030, et prenaient fin le 3 août par la commémoration de la translation de ses reliques, qui selon une légende remontant probablement au XII ${ }^{e}$ siècle et qui s'exprime notamment dans les sagas, auraient eu lieu le 3 août 1031, sous l'égide de l'évêque Grimkell, le principal compagnon missionnaire du roi Olaf. Le saint y était présenté comme le principal intercesseur auquel il convenait de s'adresser dans l'espoir d'accéder à la vie éternelle. On en prendra pour preuve la première antienne qui était chantée lors de l'office des vêpres du 28 juillet, et qui marque donc l'ouverture des cérémonies: "Sancte martyr domini olaue pro nobis quesumus apud deum intercede: ut concedat nobis delictorum veniam et vite eterne largiatur premia». L'antienne suivante appelait sur les fidèles la protection du saint en des termes très généraux: "Sancte martyr olaue tua deo placita prece nos semper et ubique protege $"^{33}$. 
Enfin, la fonction protectrice de saint Olaf était également affirmée par l'image. On sait à quel point les représentations sculptées ou peintes du saint étaient omniprésentes dans les églises du monde nordique au Moyen Âge, et les spécialistes n'ont pas manqué de souligner la fréquence d'un thème iconographique qui pourrait se rapprocher de l'idée d'une protection spirituelle. À la fin du Moyen Âge, saint Olaf est en effet souvent représenté marchant sur une autre figure, qui est soit un guerrier soit un être fantastique ${ }^{34}$. Ce type de représentation a été interprété de diverses manières, et on ne saurait certainement être trop prudent en cette matière. Il n'est néanmoins pas impossible que la figure inférieure, en particulier lorsqu'il s'agit d'un dragon, soit le symbole du mal ou du diable ${ }^{35}$. Dans ce cas on pourrait rapprocher ces représentations des deux miracles évoqués plus haut.

\section{La protection de saint Olaf et les attentes spirituelles des Norvégiens au tournant du XIIIe siècle}

Indéniablement, l'Église norvégienne de la fin du XII ${ }^{e}$ siècle et du début du XIII ${ }^{e}$ siècle invitait les fidèles à rechercher la protection de saint olaf pour lutter contre les tentations diaboliques, échapper à la damnation et accéder à la vie éternelle. Répondait-elle ainsi à une demande? Autrement dit, les Norvégiens attendaient-ils vraiment dès cette époque que leur saint roi les protégeât de cette manière?

Les $\mathrm{XI}^{\mathrm{e}}$ et XII ${ }^{\mathrm{e}}$ siècles nous ont laissé un ensemble de poèmes scaldiques en l'honneur de saint Olaf, qui permettent d'esquisser une première réponse à ces questions. Si la plupart de ces pièces ont été composées fort peu de temps après la mort du roi, comme l'Erfidrápa Óláfs helga du scalde Sighvat, qui doit dater au plus tard du début des années 1040, d'autres sont en revanche plus récentes, comme le long poème intitulé Geisli que le scalde Einarr Skúlason déclama à l'occasion des festivités qui accompagnèrent la naissance de la province ecclésiastique de Nidaros au milieu du XII ${ }^{\mathrm{e}}$ siècle. Tous ces poèmes chantent la gloire du roi martyr et les nombreux miracles de guérison qu'il opéra. Ils le dépeignent également comme un protecteur, mais force est de constater que cette fonction de protection ne s'exerce nullement dans le domaine spirituel: le rôle de saint Olaf est avant tout de protéger les rois de Norvège, qui sont ses héritiers et ses descendants. Attentif à la prospérité de son ancien royaume et garant de son intégrité, il les aide à bien le gouverner et à le protéger. Trois strophes du poème Geisli racontent ainsi comment Magnus le Bon, le fils d'Olaf Haraldsson qui régna de 1035 à 1047, bénéficia de la protection de son père au cours d'une bataille qui l'opposa aux Wendes païens au lieu dit Hlýrskógsheiðr ${ }^{36}$. Un poème plus ancien, l'óláfsdrápa, composé vers 1070 par le scalde Steinn Herdísarson en l'honneur du roi Olaf le Tranquille (1066-1093), et évoquant la lutte entre ce roi et son rival danois Sven Estridssen, a une teneur similaire ${ }^{37}$.

L'examen des sagas qui furent consacrées à saint Olaf à partir de la fin du XII ${ }^{\mathrm{e}}$ siècle et au cours du siècle suivant amène à des conclusions sensiblement identiques. Nous laisserons cependant de côté celles qui sont très tributaires du discours ecclésiastique, comme la Saga légendaire de saint Olaf, une œuvre transmise par un manuscrit norvégien du milieu du XIII ${ }^{\mathrm{e}}$ siècle et qui reprend presque mot à mot le recueil de miracles de 
l'homiliaire, pour nous concentrer sur d'autres œuvres qui se détachent plus nettement de ce discours ${ }^{38}$.

21 Des années 1190 date la rédaction dans la région du Trøndelag, c'est-à-dire la région de Nidaros, d'une compilation de sagas intitulée Àgrip af Nóregskonunga sögum. Les miracles de saint Olaf y sont évoqués de manière très rapide, et l'auteur se contente de signaler qu'ils se multiplièrent rapidement après la mort du roi $^{39}$. Il ne s'attarde quelque peu que sur un seul miracle : il s'agit à nouveau du récit de la bataille de Hlýrskógsheiðr, déjà racontée par Einarr Skúlason dans le poème Geisli, et au cours de laquelle Magnus le Bon mit en fuite les Wendes grâce au secours de son père ${ }^{40}$. L'action protectrice de saint Olaf semble dans cette compilation de sagas se limiter à cette simple dimension, ce qui est conforme à l'impression donnée par les poèmes scaldiques déjà évoqués.

On retire un sentiment similaire de la Saga de Sverre, composée par Karl Jónsson, abbé du monastère islandais de pingeyrar, en partie sous la surveillance de ce roi, qui régna entre 1177 et $1202^{41}$. Cette saga est donc largement une œuvre de propagande, destinée à affirmer la légitimité de Sverre, qui ne cesse de s'y réclamer de saint Olaf. Il est particulièrement intéressant de constater l'importance que prend dans ce discours de légitimation le thème de la protection accordée par le saint roi de Norvège à celui qui prétend être son lointain héritier. Il s'agit la plupart du temps d'une protection militaire, accordée par le saint à Sverre en vertu de la justice de sa cause. C'est donc à son intervention et à son aide qu'il attribue les victoires remportées contre Magnus Erlingsson. On retiendra ici l'exemple d'une bataille navale que les deux rois se livrèrent en 1179, et qui fait l'objet du trente-deuxième chapitre de la saga. Le rapport de forces était apparemment très défavorable à Sverre, et les bateaux de son adversaire étaient beaucoup plus rapides que les siens. Il en appela alors à saint Olaf, qui fit tomber un épais brouillard sur la mer pour le protéger ${ }^{42}$. Dans cette saga on retrouve également l'idée selon laquelle la protection de saint Olaf pouvait être recherchée pour se prémunir contre des dangers naturels. Le vingtième chapitre de l'œuvre raconte ainsi comment, en 1177, alors qu'il traversait le Sognefjell et le Vossfjell, des régions montagneuses situées dans l'ouest de la Norvège, Sverre aurait été pris dans la tempête. Jugeant qu'il s'agissait là de l'œuvre du diable, il déclara qu'il fallait s'en remettre à la miséricorde divine, et prier Dieu, la Vierge et saint Olaf. La tempête s'arrêta alors ${ }^{43}$.

Enfin, on ne saurait terminer ce rapide panorama des sagas royales du début du $\mathrm{XIII}^{\mathrm{e}}$ siècle sans évoquer les deux magistrales sagas de saint Olaf composées par l'Islandais Snorri Sturluson dans les années 1220-1230. On donne généralement à la plus longue le titre de Saga séparée de saint Olaf, pour la distinguer de l'autre, plus courte, qui fait partie intégrante du cycle de sagas royales surnommé Heimskringla, qui couvre l'histoire de Norvège de ses origines mythiques jusqu'en 1177. Toutes deux comportent globalement les mêmes récits de miracles, à ceci près que dans la Heimskringla ils sont disséminés harmonieusement dans les sagas consacrées aux successeurs d'Ola ${ }^{44}$. Chacune comporte ainsi deux récits miraculeux, ce qui matérialise en quelque sorte l'idée que tous les descendants du roi bénéficièrent de sa protection. Le choix que fit Snorri des miracles attribués à saint Olaf ne manque pas d'intérêt ${ }^{45}$. On notera en particulier que ceux-ci ne proviennent que partiellement de la collection élaborée par l'archevêque Eysteinn et de l'homiliaire. Certains sont totalement inconnus par ailleurs, et proviennent certainement de traditions orales recueillies par Snorri au cours du séjour qu'il effectua en Norvège ${ }^{46}$. Les deux sagas de Snorri donnent donc de saint Olaf une image quelque peu décalée par rapport à celle qui ressort de la légende 
ecclésiastique. On y retrouve certes les fonctions essentielles détaillées plus haut, et les miracles de guérison n'y manquent pas. Mais la fonction de protection du saint reste limitée à des aspects purement matériels et militaires, comme dans la Saga de Sverre. Il vient ainsi au secours des combattants dont la cause est juste, ce qui apparait une nouvelle fois dans le récit de la bataille de Hlýrskógsheiðr, inséré dans la Saga de Magnus le Bon (Magnúss saga ins góda), et dans celui de la victoire de l'empereur byzantin, intégré dans une des dernières sagas du cycle ${ }^{47}$. Il est également protecteur des biens de ceux qui l'invoquent, ce que l'on voit dans la Saga de Magnus berføtt (Magnúss saga berfoetts), qui reprend le récit de l'incendie de Nidaros, miraculeusement arrêté grâce à son intervention ${ }^{48}$. Enfin, il protège les hommes contre l'injustice, thème qui est illustré par la reprise dans la Saga des fils de Harald (Haraldssona saga) du récit de la guérison miraculeuse du prêtre anglais affreusement mutilé par deux Norvégiens jaloux de l'honneur de leur sœur ${ }^{49}$. Tous ces thèmes étaient déjà présents dans la Passio Olavi et dans l'homiliaire. Mais on ne retrouve nulle part chez Snorri l'idée d'une protection spirituelle.

24 Le dix-septième miracle de ces deux collections, qui développait précisément ce thème, n'eut en définitive dans les sagas qu'une postérité limitée, ce dont on peut raisonnablement conclure qu'au début du XIII ${ }^{e}$ siècle il ne correspondait pas encore vraiment aux attentes des Norvégiens. On ne le retrouve à cette époque que dans la Saga légendaire de saint Olaf. Il fut en revanche inséré beaucoup plus tard dans un manuscrit interpolé de la Saga séparée de saint Olaf de Snorri Sturluson, le Livre de Flatey (Flateyjarbók). Mais ce manuscrit islandais date de l'extrême fin du XIV siècle $^{50}$. Au XIII ${ }^{e}$ siècle prévalait donc encore une conception très traditionnelle de la protection accordée par saint Olaf aux chrétiens, et les Norvégiens ne semblent pas avoir attendu alors de saint Olaf une protection pour le salut de leur âme. La principale fonction du saint roi de Norvège dans ce domaine était d'exercer une tutelle sur ses descendants et de faire en sorte que leur règne soit bénéfique.

Cela ne signifie pas nécessairement que les Norvégiens de cette époque ne concevaient aucune inquiétude quant au sort réservé à leur âme dans l'au-delà, et qu'ils n'étaient pas en quête d'une protection spirituelle. En effet, la conception chrétienne de la mort paraît avoir assez rapidement fait souche dans le Nord, s'il faut en croire en tout cas la poésie religieuse chrétienne qui se développa en Norvège et en Islande dès le $\mathrm{XI}^{\mathrm{e}}$ siècle, empruntant à la poésie scaldique des temps païens ses règles de composition ${ }^{51}$. Le souci des âmes des morts y est assez bien attesté, comme le montrent les trois exemples qui suivent. Un scalde du milieu du XI ${ }^{e}$ siècle, Arnórr, surnommé «le scalde du jarl » (Arnórr jarlaskáld), a composé quelques strophes qui sont des prières dans lesquelles il recommande à la miséricorde divine l'âme d'un défunt ${ }^{52}$. On lui attribue également la composition d'une strophe isolée (vísa), qui évoque le Jugement dernier et la figure de saint Michel ${ }^{53}$. Enfin on a conservé une strophe attribuée à l'un des tout premiers poètes chrétiens de Scandinavie, Hallfred, surnommé le Scalde ombrageux (Hallfrøðr vandrœðaskáld), qui aurait été selon la tradition converti par le roi Olaf Tryggvason à la fin du $\mathrm{X}^{\mathrm{e}}$ siècle ${ }^{54}$. En réalité, cette vísa, transmise par l'auteur islandais de la saga qui fut consacrée à ce scalde vraisemblablement au début du XIII ${ }^{\mathrm{e}}$ siècle, pourrait fort bien ne pas être authentique et avoir été composée par l'auteur de la saga lui-même ${ }^{55}$. Elle est la seule strophe scaldique conservée qui trahisse une véritable peur de l'enfer: le scalde, sentant sa mort venir, craint pour son âme parce qu'il a beaucoup péché pendant sa jeunesse ${ }^{56}$. Il est assez difficile de tirer des enseignements définitifs de ces quelques pièces poétiques. Elles reflètent certes moins le discours de l'Église que la manière dont 
il pouvait être perçu par une partie des fidèles, et constituent par conséquent une source de renseignements assez précieuse pour qui s'intéresse aux mentalités religieuses des gens du Nord au Moyen Âge. Mais elles ne représentent peut-être que le point de vue d'une élite, et rien ne permet a priori de leur conférer une valeur universelle. Il serait donc quelque peu aventureux d'y voir le reflet des attentes et des craintes de la population norvégienne dans son ensemble, et d'en conclure que le besoin de sécurité spirituelle était très répandu dans la Norvège des alentours de 1200 . Certains en tout cas devaient le ressentir, mais il est clair que pour l'apaiser ils ne se tournaient pas vers saint Olaf. L'exemple suédois laisse penser qu'ils s'adressaient plutôt à d'autres protecteurs, comme saint Michel ou la Vierge ${ }^{57}$.

Pourquoi dans ces conditions l'Église norvégienne de la fin du XII ${ }^{e}$ siècle a-t-elle voulu donner de saint Olaf l'image d'un protecteur spirituel ? Le prologue qui précède le dixseptième miracle de la collection de miracles dans la Passio et miracula beati Olavi comme dans l'homiliaire vieux-norvégien permet peut-être d'avancer une hypothèse à ce sujet $^{58}$. Il s'agit d'un paragraphe assez court, très simple par sa teneur et à la tonalité très didactique, qui constitue une manière d'introduction au récit miraculeux et qui en souligne d'emblée la portée. On y lit que l'âme d'un croyant est beaucoup plus précieuse que son corps, et que par conséquent la guérison d'une âme a plus de valeur que celle d'un corps. Le diable, cet «ennemi du genre humain» (uvínr allz mankyns), cherche quotidiennement à tenter les hommes et à empêcher le bien de prendre racine en leur cœur ; de même qu'à l'origine des temps il a détourné Adam du droit chemin, causant le malheur de ses descendants, il cherche sans relâche à corrompre l'homme, lui faisant miroiter les honneurs temporels pour mieux lui faire oublier les châtiments éternels. Ce discours très simple s'inscrit très nettement dans une logique de christianisation de la population norvégienne; on y retrouve des thèmes fréquents dans les homélies anglo-saxonnes, dont tout laisse penser qu'elles avaient inspiré le discours des premiers missionnaires. L'objectif de l'Église, en suscitant l'idée d'une protection contre les tentations diaboliques assurée par un saint éminemment populaire comme saint Olaf, devait vraisemblablement être d'ancrer plus profondément dans les mentalités l'idée du péché et la nécessité de s'en garder pour accéder à la vie éternelle.

\section{Saint Olaf, patron des guildes et protecteur des âmes}

Cette entreprise fut apparemment couronnée d'un certain succès. C'est ce qui semble ressortir en tout cas des statuts de guildes norvégiennes datant de la fin du Moyen Âge. Ces institutions, très répandues dans toute la Scandinavie à cette époque, constituaient des associations de laïcs urbains ou ruraux, qui se rattachaient d'une certaine manière à d'anciennes solidarités remontant aux temps païens, à l'exemple de ces associations appelées omgangsdrikkelag et fondées sur le partage de la boisson ${ }^{59}$. Au terme d'une évolution que la rareté des sources ne permet pas de décrire avec précision, elles devinrent à la fin du Moyen Âge des associations garantissant à leurs membres une sécurité que la société traditionnelle n'était plus en mesure de leur fournir ${ }^{60}$. Il est donc possible d'y lire en creux les peurs et les attentes des Norvégiens de cette époque. Trois statuts de guildes ont été conservés pour la Norvège médiévale ${ }^{61}$. Le plus ancien, qui est celui d'une guilde du Trøndelag, nous a été transmis par un manuscrit du milieu du XIII ${ }^{e}$ siècle mais fut certainement élaboré avant 1200 . Les deux autres statuts, qui nous intéressent tout particulièrement parce qu'ils sont ceux de guildes placées sous le 
patronage de saint Olaf, datent respectivement du début et de la fin du XIV siècle; le premier provient du Gulatingslag, la région occidentale de la Norvège, tandis que le second, celui de la guilde d'Onarheim, provient du Sunnhordland et est daté précisément de $1394^{62}$. Les chercheurs qui s'y sont intéressés considèrent que ces statuts traduisent une évolution non seulement de l'organisation des guildes au cours des XIII ${ }^{\mathrm{e}}$ et XIV ${ }^{\mathrm{e}}$ siècles, mais aussi une évolution de leurs objectifs.

Le statut de la guilde du Gulating, qui remonte aux alentours de 1300, place cette institution sous le patronage de Jésus-Christ, de la Vierge et de saint Olaf ${ }^{63}$. Elle apparaît d'abord comme une association de sécurité mutuelle, puisque ses membres s'engageaient à maintenir la paix en son sein, à ne pas avoir recours à la violence et à venir désarmés aux réunions ${ }^{64}$. Elle constituait donc peut-être une réponse à l'insécurité latente en Norvège à la fin du Moyen Âge, récemment mise en valeur dans une étude de Jørn Sandnes ${ }^{65}$. La solidarité jouait en cas de catastrophe touchant les biens d'un membre de la guilde. Des articles prévoyaient ainsi un système de compensation en cas de destruction par le feu d'une grange ${ }^{66}$. Elle jouait également en cas de maladie du bétail ${ }^{67}$. Au cas enfin où l'un des membres de la guilde était fait prisonnier à l'étranger, ses confrères devaient contribuer financièrement à sa libération ${ }^{68}$.

Ces quelques points reflètent à l'évidence des craintes qui étaient déjà celles des Norvégiens du XII ${ }^{e}$ siècle, telles qu'en tout cas elles apparaissent au travers des miracles attribués à saint Olaf dans la Passio Olavi ou dans l'homiliaire vieux-norvégien. Mais désormais leur prise en charge n'était plus confiée à la seule intercession du saint mais était assumée par la communauté des membres de la guilde. Est-ce à dire que saint Olaf ne jouait plus son rôle de protecteur auprès des Norvégiens? Ce n'est pas du tout l'impression que donnent les derniers articles du statut, réglant les dispositions à suivre en cas de décès d'un membre de la guilde. À titre d'illustration, le trentetroisième article prévoyait qu'au cas où une sœur de la guilde viendrait à mourir, tous les frères en ayant reçu la nouvelle devaient suivre sa dépouille jusqu'à sa tombe et faire dire une messe des morts pour son âme ${ }^{69}$. Indépendamment de cela, des messes des morts devaient être chantées lors des réunions annuelles de la guilde ; l'assistance y était obligatoire sous peine d'amende ${ }^{70}$. Ces messes, destinées à assurer le salut de tous les frères et sœurs de la guilde, étaient chantées en l'honneur de Dieu et du saint roi Olaf ${ }^{71}$.

La principale protection demandée à saint Olaf, à en lire les statuts de cette guilde, était donc désormais de nature spirituelle; si les hommes prenaient en charge les questions d'assistance matérielle, ils s'en remettaient entièrement à lui à l'heure de leur trépas, et l'importance des messes des morts montre qu'ils attendaient son secours au moment du passage vers l'au-delà. Le dernier article du statut en est la claire illustration : «Que Dieu et saint Olaf accordent force et secours à ceux qui seront garants de ces lois, pour la paix et la prospérité, pour le bonheur en ce monde et pour l'entrée dans le royaume des cieux pour l'éternité. Amen ${ }^{72}$ ».

31 L'étude du statut d'Onarheim, daté de 1394, permet d'aboutir à des conclusions similaires, à ceci près que la dimension spirituelle de la guilde est beaucoup plus affirmée encore. Les dispositions relatives à la sécurité et à la paix en son sein sont présentes, mais l'essentiel des articles est consacré à la mort et aux obligations des frères et des sœurs en cas de décès de l'un des leurs. Fait significatif de cette inflexion, tous devaient payer annuellement une cotisation appelée saala skoth, ce qui signifie 
littéralement «taxe pour l'âme $»^{73}$. Et l'association, qui se réunissait pour la fête de saint Olaf, se trouvait encore plus résolument que la précédente placée sous l'égide et la protection de ce saint. Tout membre entrant ou sortant de la salle de la guilde devait s'incliner devant son image, sous peine d'amende ${ }^{74}$. Chaque année était chantée une messe solennelle le jour de la saint Olaf, à laquelle devaient participer tous les membres de la guilde ${ }^{75}$. Pendant les quelques jours où les membres de la guilde se retrouvaient, ils devaient lire chaque soir les noms de tous les frères et sœurs vivants après une prière à Marie ; puis, après une prière à saint Olaf, on lisait la liste de tous les membres de la guilde, avec requiem et de profundis ${ }^{76}$. Le dernier jour on devait chanter une messe des morts pour les âmes de tous les chrétiens, et en particulier pour les membres de la guilde $^{77}$. Enfin, en cas de décès de l'un d'entre eux, les frères et sœurs habitant dans la même paroisse que lui devaient assister à ses funérailles, faire chanter une messe des morts pour lui et fournir le luminaire ${ }^{78}$.

Les préoccupations d'ordre spirituel sont donc prédominantes dans le statut d'Onarheim et le salut des membres de la guilde s'y trouve résolument placé sous la protection de saint Olaf. Cette inflexion est certainement à lier à l'énorme mortalité entraînée en Norvège par le déferlement de la peste noire, dont on ne peut véritablement mesurer l'impact démographique, mais qui fut certainement considérable, si ce n'est même plus important en proportion que dans des pays situés plus au sud ${ }^{79}$. Il est en tout cas certain que par réflexe contre le déferlement de ces malheurs la collectivité norvégienne se tourna résolument vers saint olaf, considéré non plus tant comme le protecteur du roi mais comme celui du royaume tout entier. On en trouvera la preuve dans un acte royal du 26 avril 1365, dans lequel le roi Håkon VI s'adresse aux habitants du diocèse d'Oslo pour leur recommander d'invoquer la miséricorde de saint Olaf et de saint Hallvard en ces temps de malheur ${ }^{80}$.

Ce rapide panorama des sources norvégiennes des derniers siècles du Moyen Âge permet de tirer quelques conclusions prudentes sur les peurs et les attentes spirituelles des Norvégiens de cette époque. Entre la fin du XII e siècle et le XIV siècle, la protection qu'ils attendaient de saint Olaf semble avoir évolué. Alors qu'il était à l'origine considéré comme le tuteur des rois et comme un saint dont le secours pouvait être recherché par quiconque craignait pour ses biens ou pour sa vie, il devint également peu à peu un protecteur des âmes, dans la perspective du salut. Il est difficile de dater très précisément cette inflexion; mais tout laisse croire qu'elle fut antérieure au déferlement de la peste en Norvège et au cortège de malheurs qui l'accompagna.

Par ailleurs, il semble que l'idée selon laquelle on pouvait attendre de saint olaf une protection spirituelle ne soit pas née spontanément en Norvège. Au contraire elle résulta d'une initiative ecclésiastique remontant à la seconde moitié du XII ${ }^{\mathrm{e}}$ siècle. Il s'agissait probablement pour la jeune Église de Nidaros d'ancrer plus profondément dans les mentalités l'idée du péché, assimilé à une tentation diabolique, et la nécessité de se prémunir contre lui dans la perspective du salut. Ce thème s'insère donc dans le long effort entrepris par les clercs de Norvège pour christianiser des populations encore très fortement marquées par les mentalités germaniques païennes et très imparfaitement christianisées. L'émergence d'un besoin de protection spirituelle en Norvège, vraisemblablement au cours du XIII ${ }^{e}$ siècle, constitue donc un indice sûr de la christianisation de ce royaume nordique, christianisation dont l'image de saint Olaf fut certainement l'un des principaux ferments. 


\section{NOTES}

1. J. Delumeau, Rassurer et protéger. Le sentiment de sécurité dans l'Occident d'autrefois, Paris, Fayard, 1989, pp. 179-260.

2. Sans aller jusqu'à accréditer la version donnée par les sagas, selon laquelle Olaf Haraldsson fut déclaré saint un an et cinq jours après sa mort, soit le 3 août 1031, tout laisse penser que sa sainteté fut établie dans les années 1030, peut-être sous le règne de son fils Magnus le Bon (1035-1047) : cf. C. Krag, Vikingtid og Rikssamling 800-1130, Oslo, Aschehoug, 1995, pp. 160-164. La diffusion du culte de saint Olaf en dehors de la Norvège a fait l'objet de nombreuses études ; on renverra en dernier lieu à la synthèse de Lars Roar Langslet, Olav den hellige,Oslo, Gyldendal, 1995, pp. 121-142, et aux importants articles publiés dans Helgonet $i$ Nidaros: Olavskult och kristnande $i$ Norden, réd. L. Rumar,Skrifter utgivna av Riksarkivet 3, Stockholm, 1997.

3. Adam de Brême, Gesta Hammaburgensis ecclesice pontificum, éd. B. Schmeidler, Monumenta Germanice Historica, Scriptores rerum Germanicarum in usum scholarum, Hanovre-Leipzig, 1917. Cette information est donnée par Adam au chapitre 33 du livre IV des Gesta (pp. 267-268).

4. L. Daae, Norges helgener, Christiania, Alb. Cammermeyers Forlag, 1879, p.61. On pourrait aisément multiplier les exemples attestant la longévité de son culte. On s'en tiendra à la demande adressée en 1316 au roi de Norvège Håkon $\mathrm{V}$ par les Dominicains de Bruges, qui avaient dédié un autel en leur église à saint Olaf et souhaitaient recevoir une image et des reliques de ce saint, ainsi que sa légende : cf. Diplomatarium Norvegicum. Oldbreve til Kundskab om Norges indre og ydre Forhold, Sprog, Slcegter, Sceder, lovgivning og rettergang i middelalderen VIII, $1, \mathrm{n}^{\circ} 48$, éd. C.R. Unger et H.J. Huitfeldt, Christiania, 1871, pp. 71-72.

5. Outre les diocèses norvégiens de Nidaros, Bergen, Stavanger, Oslo et Hamar, la province regroupait les deux diocèses islandais de Skálaholt et Hólar, ainsi que le Groenland, les Féroé, les Orcades et les Hébrides.

6. L'existence d'une translatio sancti Olavi est suggérée par l'Historia de antiquitate regum Norwagensium rédigée par le moine Theodoricus probablement dans les années 1180 : cf. G. Storm (éd.), Monumenta historica Norvegice, Christiania, 1880, pp. 43-44.

7. Le plus ancien de ces manuscrits (MS Douai 295) date des environs de 1200 et provient du monastère bénédictin d'Anchin, à proximité d'Arras : cf. L. Gjerløw, "Olav den hellige. Liturgi », Kulturhistorisk Leksikon for nordisk middelalder, vol. XII, Copenhague, 1967, pp. 561-567.

8. Une édition de ce manuscrit a été donnée à la fin du XIX ${ }^{\mathrm{e}}$ siècle par FredÉrick Metcalfe (F. Metcalfe (éd.), Passio et miracula beati Olavi : edited form a twelfth-Century manuscript in the Library of Corpus Christi College, Oxford, Clarendon Press, 1881). Cette édition étant malheureusement introuvable en France, on renverra ici à la traduction qui en a été faite par Eiliv Skard en norvégien nynorsk: Passio Olavi. Lidingssoga og undergjerningane åt den heilage Olav,trad. E. Skard, Norrøne bokverk 26, Oslo, Det Norske Samlaget, 1970.

9. E. Skard, Sprache und Stil der Passio Olavi,Avhandlinger utgitt av Det norske videnskaps-Akademi i Oslo II., Hist.-Filos. klasse, 1932 n 1, Oslo, 1932 ; A. Holtsmark, "Sankt Olavs liv og mirakler », Festskrift Francis Bull,Oslo, 1937, pp. 212-233 (repris dans Studier i norrøn digtning,Oslo, Gyldendal, 1956, pp. 15-24).Le recueil de miracles a été de toute évidence rédigé en plusieurs étapes. On distingue aisément quatre strates à l'intérieur de la collection, et seuls les neuf premiers miracles sont antérieurs au milieu du XII ${ }^{\mathrm{e}}$ siècle (cf. E. Gunnes, Erkebiskop Øystein. Statsmann og kirkebygger, Oslo, Aschehoug, 1996, pp. 208-209).

10. A. Holtsmark, Studier i norrøn digtning, op. cit., p. 24.

11. T. Knudsen, «Homiliebøger", Kulturhistorisk Lexikon for nordisk middelalder, vol. VI, Copenhague, 1961, pp. 650-664. 
12. Selon la philologue norvégienne Anne Holtsmark, les deux textesproviendraient d'une même vita antérieure perdue : cf. A. Holtsmark, Studier i norrøn digtning, op. cit., p. 22.

13. Gamal norsk homiliebok, Cod. AM $6194^{\circ}$, éd. G. Indrebø, Oslo, 1931, pp. 108-129.

14. Il s'agit du trente-huitième miracle de la collection : cf. Passio et miracula beati Olavi, op. cit., pp. 64-66.

15. Le vingtième miracle de la collection, que l'on retrouve également dans l'homiliaire et qui a pour théâtre l'église construite sur le champ de bataille de Stiklestad, où Olaf avait trouvé la mort en 1030, met ainsi en scène un roi de Norvège qui avait été blessé à la jambe. L'ayant posée sur la pierre qui se trouve sous l'autel et sur laquelle Olaf aurait expiré, il fut guéri le soir même : cf. Passio et miracula beati Olavi, op. cit., p. 50.

16. À partir d'une très vaste documentation, Pierre-André Sigal a conclu que les guérisons et résurrections représentaient $56,9 \%$ des miracles attestés dans la France des $\mathrm{XI}^{\mathrm{e}}$ et XII ${ }^{\mathrm{e}}$ siècles (cf. P. A. Sigal, L’homme et le miracle dans la France médiévale,Paris, Le Cerf, 1985, p. 289). La comparaison avec les procès de canonisation du XIII ${ }^{\mathrm{e}}$ siècle étudiés par André Vauchez est encore plus significative : les miracles de guérison y représentent $85 \%$ de l'ensemble (cf. A. Vauchez, La sainteté en Occident aux derniers siècles du Moyen Âge, d'après les procès de canonisation et les documents hagiographiques,Bibliothèque des Ecoles françaises de Rome et d'Athènes, EFR, 1981, p. 547).

17. À cet égard Christian Krötzl a fait justement remarquer que les miracles accomplis par saint Olaf loin de Nidaros étaient proportionnellement importants pour le XII ${ }^{\mathrm{e}}$ siècle ; ils représentent selon ses calculs $37 \%$ de l'ensemble (C. Krötzl, Pilger, Mirakel und Alltag, Formen des Verhaltens im skandinavischen Mittelatlter (12.-15. Jahrhundert),Studia Historica 46, Helsinki, 1994, p. 64). Les bénéficiaires de ces miracles sont loin d'être tous norvégiens: on notera par exemple que le trente-deuxième miracle de la Passio Olavi concerne des hommes originaires de Galice.

18. Cf. miracles 12 et 29 (Passio et miracula beati Olavi, op. cit.pp. 37-40, 57-58).

19. Cf. miracle 15 (Ibid., p. 43).

20. Cf. miracle 16 (Ibid., p. 44).

21. Cf. miracle 31 (Ibid.,pp. 61-62).

22. Cf. miracle 4 (Ibid.,pp. 27-28).

23. Cf. miracle 10 (Ibid.,pp. 34-35).

24. Cf. miracle 8 (Ibid.,pp. 31-32).

25. Cf. miracle 3 (Ibid., Passio et miracula beati Olavi, op. cit., pp. 25-26).

26. Cf. miracles 11 et 14 (Ibid., p. 36, 46) et miracles 27 et 35 (Ibid., p. 55, 63).

27. Cf. miracles 30 et 28 (Ibid.,pp. 59-60, 55-56).

28. Ibid.,pp. 44-45 ; Gamal norsk homiliebok, op. cit.,pp. 125-127.

29. Passio et miracula beati Olavi, op. cit., pp. 69-71.

30. Gamal norsk homiliebok, op. cit., p. 126.

31. L. Gjerløw (éd.), Libri liturgici provincice Nidrosiensis medii æevi. Bind II : Ordo Nidrosiensis ecclesice (Orðubók), Norsk historisk Kjeldeskrift-Institutt, Oslo, 1968, pp. 124-128.

32. Monumenta historica Norvegie, op. cit., pp. 228-282.

33. Ibid., p. 229.

34. F.B. Wallem et B. Irgens Larsen, Iconographia Sancti Olavi. Olavsfremstillinger i middelaldersk kunst. Del I A. Skupturfremstillinger,Det Kongelige Norske Videnskabers Selskabs Skrifter 1930, Trondheim, 1947, pp.38-92. Ces deux auteurs notent que ce dernier type de représentation domine à partir $\mathrm{du} \mathrm{XV}$ siècle.

35. À l'appui de cette interprétation les deux spécialistes cités plus haut évoquent le Speculum Ecclesice d'Honorius Augustodunensis. Voir leur discussion dans Iconographia Sancti Olavi, op. cit., pp. 248-268.

36. Finnur Jónsson (éd.), Den norsk-islandske Skjaldedigtning,Copenhague, Gyldendal, vol. B, 1908, pp. 434-435.

37. Ibid., p. 381. 
38. Pour la saga légendaire, cf. A. Heinrichs, "Oláfs saga helga", Medieval Scandinavia. An Encyclopedia,dir. P. Pulsiano et K. Wolf,New-York-Londres, 1993, pp. 446-448.

39. Àgrip af Nóregskonunga sogum. Fagrskinna. Nóregs konunga tal,éd. Bjarni Einarsson, Íslenzk Fornrit 29, Reykjavík, 1985, chapitre 33, p. 32.

40. Ibid.,chapitre 37, p. 35.

41. Seule la première partie de la saga fut écrite sous le contrôle de Sverre. Cette partie raconte le destin du roi jusqu'en 1178. La deuxième partie fut composée ultérieurement, mais toujours par le même abbé : cf. L. Holm-Olsen, "Sverris saga », Medieval Scandinavia, op. cit.,pp. 628-629; S. Bagge, «La Sverris saga, biographie d'un roi de Norvège », Proxima Thulé 2, 1996, pp. 113-128.

42. Sverris saga etter Cod. AM 327 4to,éd. G. Indrebø, Christiania, 1880, chapitre 32, pp. 22-23.

43. Ibid.,chapitre 20, pp. 34-35.

44. Un seul miracle ne se trouve que dans la Heimskringla, précisément au quatorzième chapitre de la Saga de Harald le Sévère. Le saint aurait libéré son demi-frère qui était retenu prisonnier à Byzance.

45. D. Whaley, « Heimskringla and its sources : the miracles of óláfr helgi », The Sixth International Saga Conference, 28.7-2.8.1985 : Workshops Papers, Copenhague, 1985,volume 2, pp. 1083-1103.

46. On prendra ici l'exemple d'un miracle inséré par Snorri dans la Saga d'olaf le Tranquille (óláfs saga kyrra, chapitre 7). Au cours d'une procession, la châsse contenant les reliques du saint se fit si lourde qu'on ne put plus la déplacer. On la déposa alors et on creusa à cet endroit pour voir ce qui empêchait la procession de suivre son cours. On trouva alors le cadavre d'un enfant assassiné (cf. Snorri Sturluson. Heimskringla III, éd. Bjarni Aðalbjarnarson, Íslenzk Fornrit 28, Reykjavík, 1951, p. 209).

47. Le premier de ces miracles se trouve dans la Saga de Magnus le Bon (Magnúss saga ins góða) : ibid.,chapitres 27-28, pp. 43-45. On lira le second dans la Saga de Håkon herdebrei (Hákonar saga herðibreiðs) : ibid., chapitre 21, pp. 371-372.

48. Ibid., p. 232.

49. Ibid., pp. 334-337.

50. Saga Óláfs konungs hins helga. Den Store saga om Olav den Hellige, efter pergamenthåndskrift $i$ kungliga biblioteket $i$ Stockholm $n^{\circ} 2$ 4to med varianter fra andre hånskrifter,éd. O. A. Johnsen et Jón Helgason, Oslo, 1941, volume 2, pp. 1052-1053.

51. W. Lange, Studien zur christlichen Dichtung der Nordgermanen 1000-1200,Palaestra. Untersuchungen aus der deutschen und englischen Philologie und Literaturgeschichte 222, Göttingen, 1958.

52. Diana Edwards, qui a proposé une étude d'ensemble de l'œuvre poétique d'Arnorr, a relevé cinq prières de ce genre. Cf. D. Edwards, «Christian and Pagans references in eleventh-Century norse poetry: the case of Arnorr jarlaskáld», Saga-Book of the Viking Society,21, 1982-1893, pp. 34-53.

53. Id., The Poetry of Arnórr jarlaskáld. An Édition and Study, Turhout, Brepols, 1998, p. 134. Il s'agit d'un poème funéraire daté approximativement de 1073.

54. D'après la tradition des sagas, Hallfred se convertit au christianisme sous l'influence du roi Olaf Tryggvason, qui régna à la fin $\mathrm{du} \mathrm{X}^{\mathrm{e}}$ siècle et qui fut l'un des principaux rois missionnaires de Norvège. On peut renvoyer par exemple à la saga que consacra à ce roi Snorri Sturluson dans le cycle de la Heimskringla: cf. Snorri Sturluson, Histoire des rois de Norvège. Première partie. Des origines mythiques de la dynastie à la bataille de Svold,trad. F.X. Dillmann, Paris, Gallimard, 2000, pp. 318-319.

55. Bjarni Einarsson, «The Last hour of Hallfreðr vandræðaskáld as described in Hallfreðarsaga », Proceedings of the Eigth Viking Congress, Århus 24-34 August 1977, éd.H. Bekker-Nielsen, P. Foote, Odense University Press, 1981, pp. 217-221.

56. En voici la traduction proposée par Régis Boyer (cf. La Saga de Gunnlaug langue-de-serpent et la Saga de Hallfredr le scalde difficile, trad. R. Boyer, Paris, 1998, p. 120) : 
Je devrais maintenant mourir

sans souci si je savais

mon âme sauvée ;

jeune, j'avais la langue acérée ;

je sais que je ne crains rien

- chacun doit mourir -

si ce n'est que j'ai peur de l'enfer ;

Dieu décide où je vivrai dans l'au-delà.

57. Nous renvoyons ici au poème d'Arnórr cité plus haut, ainsi qu'à des inscriptions runiques suédoises relevées par Sven Jansson. L'une d'entre elles, retrouvée à Bornholm, appelle précisément la protection de saint Michel sur les âmes de deux défunts : "Que Dieu et saint Michel aident les âmes d'ödhbjörnr et de Gunhildr à entrer dans la lumière du Paradis !» (S.B.F. Jansson, Runinskrifter $i$ Sverige,Stockholm, 1963, p.119; traduction dans M. Gravier, Les Scandinaves. Histoire des peuples scandinaves, épanouissement de leurs civilisations des origines à la Réforme, Lidis-Brepols, Paris, 1984, p. 314). Une autre inscription retrouvée dans l'Uppland fait appel à la protection de la Vierge : «Ulfketill et Gyi ont fait dresser cette pierre à la mémoire d'Ulfr, leur excellent père. Il était établi à Skulhambr. Que Dieu et la mère de Dieu viennent en aide à son esprit et à son âme, qu'ils lui accordent la lumière et le Paradis " (S.B.J. Jansson, Runinskrifer $i$ Sverige, op. cit. p. 113; traduction dans M. Gravier, Les Scandinaves, op. cit. pp. 313-314).

58. Passio et miracula beati Olavi, op. cit., p. 44 ; Gamal norsk homiliebok, op. cit., pp. 125-126.

59. G. A. Blom, «Gilde», Kulturhistorisk Leksikon for nordisk middelalder,volume V, 1960, pp. 308-313.

60. H. Bjørkvik, Folketap og sammenbrudet 1350-1520, Oslo, Aschehoug, 1996, pp. 114-118.

61. Ils ont été édités à la fin du XIX ${ }^{\mathrm{e}}$ siècle par Gustav Storm (Norges gamle love, éd. G. Storm, Kristiania, 1895, volume 5, pp. 7-13) ; on renverra également à la traduction norvégienne qui en a été donnée en 1920 par l'historien Oscar Albert Johnsen (O. A. Johnsen, Tre Gildeskraaer fra middelalderen. Overscettelse fra oldnorsk med oplysninger og en inledning om gildevcesenets oprindelse og utvikling,Kristiania, 1920). L'existence d'un grand nombre d'autres guildes est avérée, même si malheureusement leurs statuts ont été perdus. Saint olaf devait être le patron d'un certain nombre d'entre elles; la chose est en tout cas assurée pour la guilde de Tønsberg, qui remontait au XIII siècle, comme l'indique la loi urbaine du roi Magnus le Législateur (1263-1280).

62. C. Anz, Gilden im mittelalterlichen Skandinavien,Veröffentlichungen des Max-Planck-Instituts für Geschichte 139, Göttingen, 1998, pp. 83-102.

63. Norges gamle love, op. cit, article 1, p. 7.

64. Ibid., articles 15 et 18, pp. 8-9.

65. J. Sandnes, Kniven, ølet og aren: kriminalitet og samfunn i Norge på 1500-og 1600 tallet, Oslo, Universitetsforlaget, 1990.

66. Norges gamle love, op. cit., articles 27 et 28, p. 9.

67. Ibid., article 29, p. 9.

68. Ibid.,article 31, p. 10.

69. Ibid., article 33, p. 10.

70. Ibid.,article 41, p. 10.

71. Ibid., article 45, pp. 10-11.

72. Norges gamle love, op. cit., article 46, p. 11.

73. Ibid., article 2, p. 11.

74. Ibid., article 18, p. 12.

75. Ibid.,article 9, p. 11.

76. Ibid.,article 12, p. 12.

77. Ibid., article 10, p. 12. 
78. Ibid., article 21, p. 12.

79. La Norvège aurait perdu entre le tiers et la moitié de ses habitants entre le milieu du $\mathrm{XIV}^{\mathrm{e}}$ siècle et le début $\mathrm{du} \mathrm{XVI}^{\mathrm{e}}$ siècle: cf. O.J. Benedictow, Plague in the late medieval nordic countries : epidemiological studies,Oslo, Middelalderforlaget, 1992.

80. DN IV, $1, n^{\circ} 448$, p. 346.

\section{AUTEUR}

\section{STÉPHANE COVIAUX}

Université d'Orléans 